\title{
A compact and versatile grating spectrograph for soft X-ray emission analysis
}

\author{
Cite as: Rev. Sci. Instrum. 90, 106105 (2019); doi: 10.1063/1.5118216 \\ Submitted: 2 July 2019 • Accepted: 19 September 2019 • \\ Published Online: 4 October 2019
}

\author{
I. Mantouvalou, , ${ }^{1, a)}$ (iD S. Staeck, ${ }^{1}$ A. Jonas, ${ }^{1}$ D. Grötzsch, ${ }^{1}$ M. Spanier, J. Baumann, ${ }^{1}$ K. Witte, \\ R. Unterumsberger, ${ }^{2}$ (D) M. Müller, ${ }^{2}$ (D) and B. Kanngießer
}

\author{
AFFILIATIONS \\ ${ }^{1} T$ U Berlin, Analytical X-Ray Physics, 10623 Berlin, Germany \\ ${ }^{2}$ Physikalisch-Technische Bundesanstalt (PTB), X-Ray Spectrometry, 12489 Berlin, Germany \\ a)ioanna.mantouvalou@tu-berlin.de \\ ${ }^{\text {b) }}$ Current address: PSI, Villigen, Switzerland.
}

\begin{abstract}
A flexible spectrograph for the investigation of soft X-ray spectra in the range between $1 \mathrm{~nm}$ and $20 \mathrm{~nm}$ is presented. Based on two variableline spaced reflection gratings, the design enables stand-alone operation, the change between the use of an entrance slit and a slitless operation mode as well as a maximum compactness and robustness due to a reduced number of motorized stages. Characterization measurements as well as first proof-of-principle X-ray emission investigations show the potential of the instrument for versatile applications.
\end{abstract}

Published under license by AIP Publishing. https://doi.org/10.1063/1.5118216

Commercial variable line-spaced (VLS) reflection gratings are widely used for X-ray emission analysis in the soft X-ray range. The most prominent characteristic of these gratings is the possibility for flat-field detection because a certain wavelength range is focused on one plane. A standard CCD deployed as the detector minimizes the number of motorized stages in a spectrograph and enables the collection of a spectrum in one shot for pulsed sources. Therefore, VLS spectrographs have been presented as compact, easy-to-use, flexible instruments with moderate resolving power.

The design of the standard VLS gratings necessitates grazing incidence geometries. All geometric parameters are determined by the used grating, requiring adapted spectrographs for each grating. For versatile applications, different requirements are apparent such as compactness, the possibility for stand-alone operation (vacuum tightness), grating holder adaption due to the geometric requirements of different gratings, and the possibility to switch between slitless design resulting in maximal efficiency and a design using different sizes of slits for maximum resolving power.

We present the design and characterization of a spectrograph based on commercial VLS gratings in the wavelength range between $1 \mathrm{~nm}$ and $20 \mathrm{~nm}(80 \mathrm{eV}-1200 \mathrm{eV})$. We demonstrate that all of the above mentioned requirements can be fulfilled in one instrument while at the same time reducing the number of motorized stages. For characterization purposes, experiments using a laser-produced plasma (lpp) source, ${ }^{2}$ as well as synchrotron radiation were conducted and soft $\mathrm{X}$-ray emission investigations exemplify the capabilities of lab-based spectrometry.

The compact design of the spectrograph (max. $300 \mathrm{~mm} \times$ $400 \mathrm{~mm} \times 600 \mathrm{~mm}$ ), see Fig. 1 , can be used in a slitless mode in a high vacuum (HV) chamber as well as with a slit system (1) for other purposes. Two variable line-spaced gratings (blue) for the wavelength range $1-5 \mathrm{~nm}$ and 5-20 $\mathrm{nm}$ are available which enable flat-field detection with a CCD camera (6), see the supplementary material for details. A manual replacement is necessary when switching between wavelength ranges. The gratings can be tilted with a motorized stage in order to switch between the imaging of the zeroth and first order of diffraction for alignment purposes. The CCD is adjustable in height with a traveling range of about $2 \mathrm{~mm}$ due to the use of a vacuum bellows for fine alignment purposes. The geometric properties of the two gratings vary in the necessary angles; therefore, the height position difference of the CCD camera is compensated by an asymmetrical flange which can be rotated by $180^{\circ}$.

The acquisition time is controlled by an electrical shutter (3). An additional motor is used to drive a translation stage (2) in front of the spectrograph for three operation modes: the spectrograph can be sealed vacuum tight and a filter $(200 \mathrm{~nm} \mathrm{Al}$ or Ti) or a pinhole can be placed in the beam path. A wire cross is used to check the alignment when observing the zeroth order. The slit system can be attached 


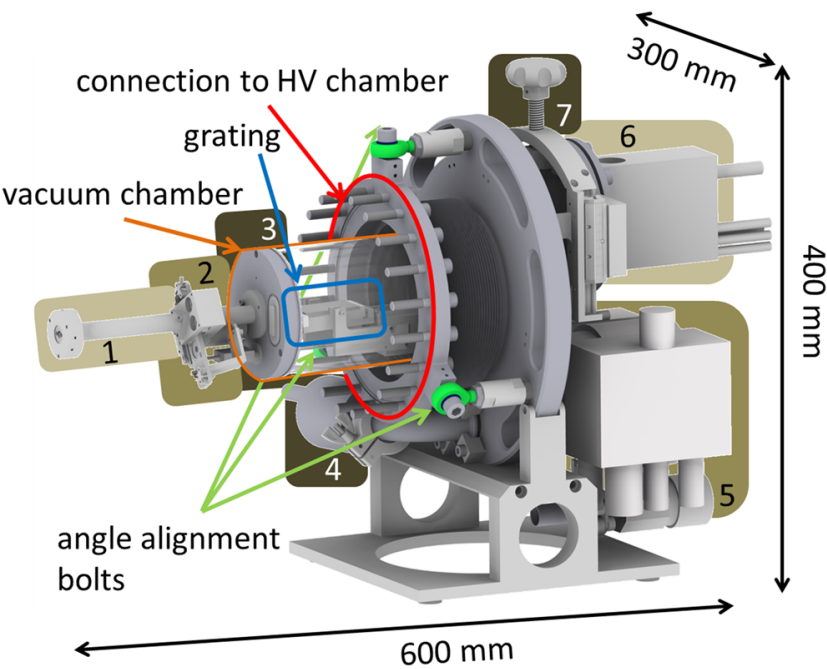

FIG. 1. Schematic CAD drawing of the spectrograph with (1) detachable snout with slit system and cross hair, (2) motor stage for three modes (pinhole, vacuum tight, and filter), (3) vacuum part with electrical feedthroughs and shutter (not visible), (4) gauge head, (5) turbomolecular pump, (6) CCD camera,and (7) height adjustment stage for the CCD.

as a snout in front of this device. A vacuum pump system (4) and (5) is attached, which allows in combination with a small vacuum chamber (orange) in sealed mode a completely independent operation of spectrograph and the surrounding vacuum system. With the help of three threaded bars (green), the direction of the spectrograph axis can be changed in respect to a $160 \mathrm{~mm}$ flange (red), which can be attached, e.g., to a vacuum chamber. This alignment mechanism facilitates the omission of at least 3 motorized stages, which are commonly used for positioning.

As a VLS grating is used, the obtained image on the CCD is a rectangle with the width determined by the source/slit size, the grating size, and the geometrical distances and the height being the dispersion direction. A spectrum is obtained by summing the intensities as a function of position in the dispersion axis. Due to the higher relevance for soft X-ray experiments, the following results will be demonstrated with the $1 \mathrm{~nm}-5 \mathrm{~nm}$ VLS grating, only.

The resolving power of the spectrograph with a $100 \mu \mathrm{m}$ slit was derived from measurements of the emission of an lpp source, which emits broadband radiation in the soft X-ray range. ${ }^{2}$ The relaxation lines from an lpp are, if they are separated, lifetimebroadened only and thus a good measure for the resolving power of the instrument. Figure 2, bottom, shows the emission spectrum of a copper plasma measured with the spectrograph. After energy calibration with the well-known relaxation lines (see, e.g., Ref. 3), fully separated lines were fitted with Gaussian curves, and the FWHM yields the resolving power. The resolving power lies between $\mathrm{E} / \Delta \mathrm{E}=150$ at $\mathrm{E}=1400 \mathrm{eV}(1 \mathrm{~nm})$ and $\mathrm{E} / \Delta \mathrm{E}=450$ at $\mathrm{E}=200 \mathrm{eV}$ $(5 \mathrm{~nm})$ for a $100 \mu \mathrm{m}$ slit or source.

The efficiency of the spectrograph was characterized using monochromatic synchrotron radiation. The spectrograph was attached to a spectroscopy chamber ${ }^{4}$ at the plane grating monochromator beamline of the PTB laboratory at the synchrotron radiation facility BESSY II.

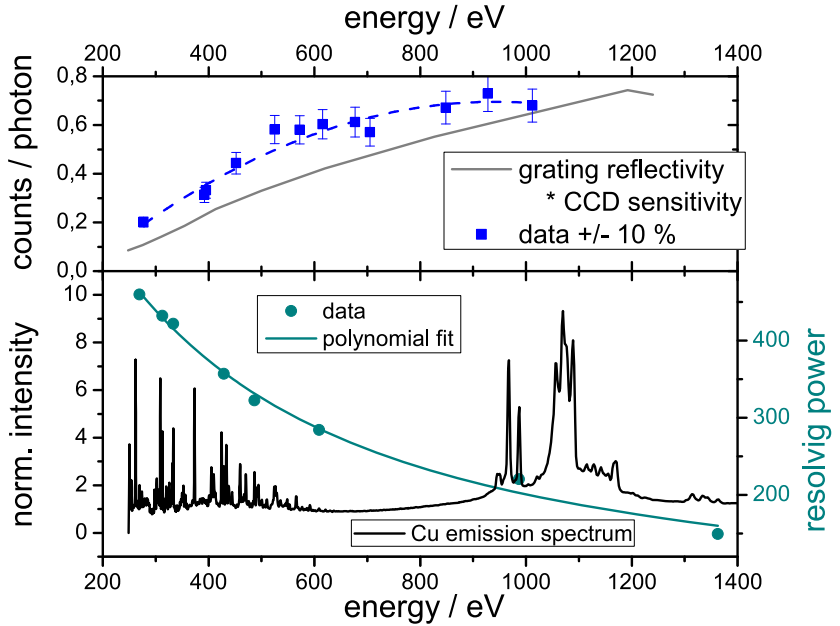

FIG. 2. Top: efficiency of the spectrograph in counts/photon (blue scatter) and simplified calculation using known grating reflectivity and CCD efficiency. Bottom: resolving power of the spectrograph with a $100 \mu \mathrm{m}$ entrance slit derived from the emission of a Cu plasma spectrum.

For the characterization experiments, a set of 10 reference samples (see the supplementary material) was measured in a $90^{\circ}$ geometry fluorescence mode with the spectrograph in slitless mode as detector. In Fig. 3, the measurement of a metallic $\mathrm{Cu}$ sample is presented as example, illustrating the superior resolving power of the spectrograph compared to silicon drift detector (SDD) detectors as well as the reduced efficiency.

Due to the fully calibrated setup of PTB, an efficiency calibration is feasible. For this purpose, the measurements were normalized to the measured fluorescence of a second calibrated SDD. The obtained efficiency of the spectrograph is plotted in the top part of

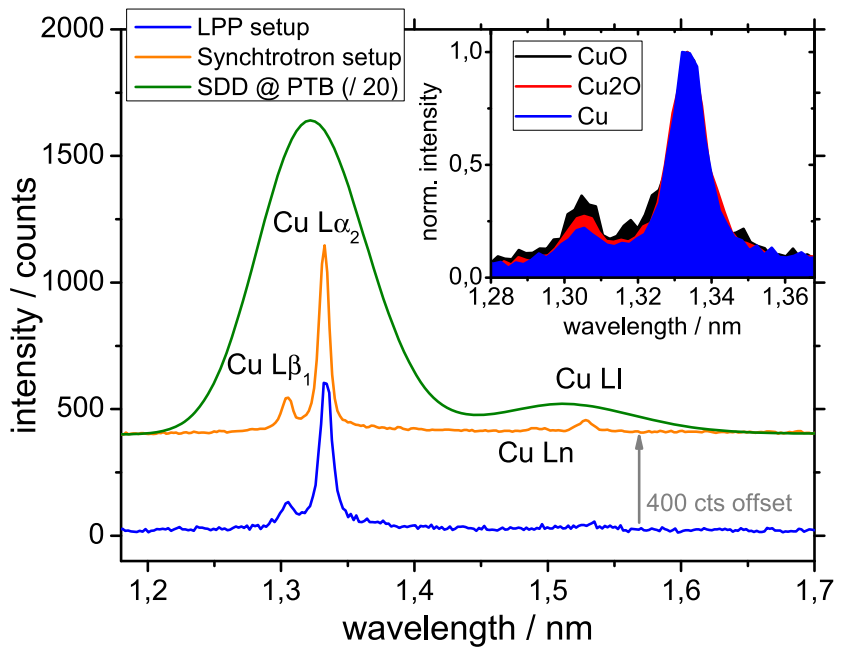

FIG. 3. Measurement of a metallic copper sample with the spectrograph and a calibrated SDD detector at PTB with $1060 \mathrm{eV}$ excitation energy and with the Ipp setup at BLiX. The inset shows the measurement of three different $\mathrm{Cu}$ species obtained with the Ipp setup. 


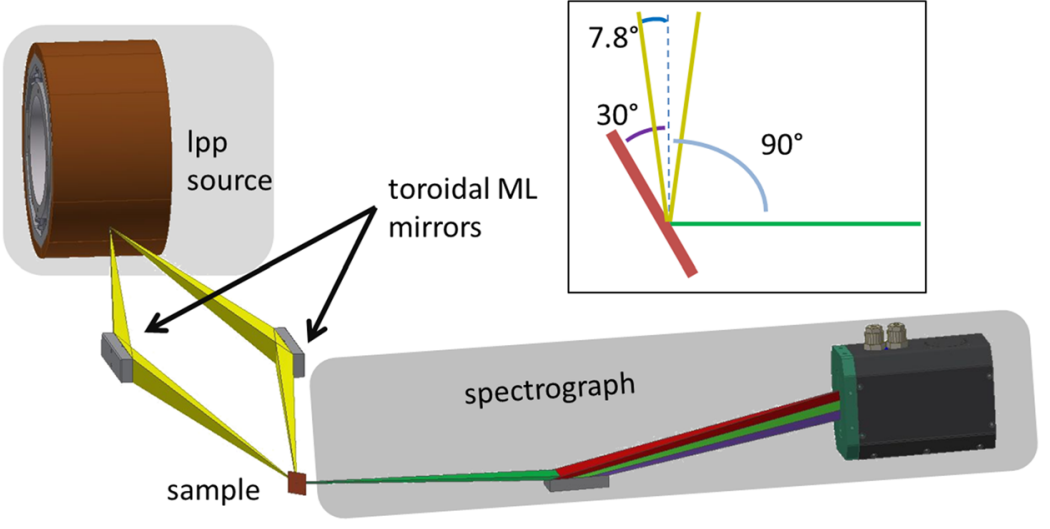

FIG. 4. Schematic CAD drawing of the XES experiment. The inset shows the excitation and detection angles.

Fig. 2 with the blue dashed line as guide for the eye and in black a simplified calculation, see the supplementary material for details.

As an application example, laboratory soft X-ray emission spectroscopy (XES) was chosen. A setup consisting of a lpp source, ${ }^{2}$ toroidal multilayer (ML) mirrors for the focusing and monochromatizing of the radiation and the spectrograph as detector was built up; see Fig. 4 and the supplementary material for details.

Three copper samples were available for proof-of-principle investigations, metallic copper, and two pressed pellets of $\mathrm{CuO}$ and $\mathrm{Cu} 2 \mathrm{O}$. Figure 3 shows the comparison of XES spectra of metallic copper obtained from the measurements at the PTB beamline with the spectrograph and with the presented setup. There is an excellent agreement of the line ratios and energetic positions between the two measurements with the spectrometer. There is a slight degradation of the resolving power between synchrotron and lpp spectra $(132 / 101 \pm 5)$ at the La line, which can be explained by the different excitation spot sizes. The possibility to distinguish different $\mathrm{Cu}$ species is demonstrated in the inset of Fig. 3. As shown in Ref. 5, the ratio of the $L \beta$ to La fluorescence intensity increases with a higher oxidation state. Despite the low statistics of our measurements, this trend can be clearly observed.

Current high-end state-of-the-art systems at large scale facilities either use two optical elements (curved mirror plus plane grating $)^{6}$ or specially designed $2 \mathrm{D}$ gratings ${ }^{7}$ in combination with long detection arms. ${ }^{8}$ While these systems exhibit larger resolving powers (up to $\mathrm{E} / \Delta \mathrm{E}=10000$ ), the efficiency is generally lower or comparable at best. We present a highly compact and robust instrument for soft X-ray emission spectroscopy with minimal motorization which is transportable and can easily be adapted to measurements with different excitation sources. Different gratings as well as operation with or without entrance slit render the flexible instrument into a versatile, well-characterized tool. Application possibilities include not only the presented plasma diagnostics and X-ray emission spectroscopy but also, e.g., simultaneous X-ray absorption spectroscopy at multiple absorption edges with moderate resolution $(\mathrm{E} / \Delta \mathrm{E}=150-450)$. Due to the simultaneous detection of the whole spectrum, it is additionally well suited for pulsed sources and thus dynamic investigations.

See the supplementary material for the technical specifications of the components, measurement details for the characterization at PTB and the XES experiment, and the alignment procedure of the spectrograph.

\section{REFERENCES}

${ }^{1}$ R. R. Fäustlin, U. Zastrau, S. Toleikis, I. Uschmann, E. Förster, and T. Tschentscher, J. Instrum. 5, P02004 (2010); T. Harada, K. Takahashi, H. Sakuma, and A. Osyczka, Appl. Opt. 38, 2743 (1999).

${ }^{2}$ I. Mantouvalou, K. Witte, D. Grötzsch, M. Neitzel, S. Günther, J. Baumann, R. Jung, H. Stiel, B. Kanngiesser, and W. Sandner, Rev. Sci. Instrum. 86, 035116 (2015).

${ }^{3}$ A. Kramida, Y. Ralchenko, J. Reader, and NIST ASD Team, NIST Atomic Spectra Database (version 5.4), available at http://physics.nist.gov/asd.

${ }^{4}$ J. Lubeck, B. Beckhoff, R. Fliegauf, I. Holfelder, P. Hönicke, M. Müller, B. Pollakowski, F. Reinhardt, and J. Weser, Rev. Sci. Instrum. 84, 045106 (2013).

${ }^{5}$ J. Kawai, K. Nakajima, and Y. Gohshi, Spectrochim. Acta 48, 1281-1290 (1993).

${ }^{6}$ O. Fuchs, L. Weinhardt, M. Blum, M. Weigand, E. Umbach, M. Bär, C. Heske, J. Denlinger, Y.-D. Chuang, W. McKinney, Z. Hussain, E. Gullikson, M. Jones, P. Batson, B. Nelles, and R. Follath, Rev. Sci. Instrum. 80, 063103 (2009).

${ }^{7}$ Z. Yin, H. Löchel, J. Rehanek, C. Goy, A. Kalinin, A. Schottelius, F. Trinter, P. Miedema, A. Jain, J. Valerio, P. Busse, F. Lehmkühler, J. Möller, G. Grübel, A. Madsen, J. Viefhaus, R. E. Grisenti, M. Beye, A. Erko, and S. Techert, Opt. Lett. 43, 4390-4393 (2018).

${ }^{8}$ G. Ghiringhelli, A. Piazzalunga, C. Dallera, G. Trezzi, L. Braicovich, T. Schmitt, V. N. Strocov, R. Betemps, L. Patthey, X. Wang, and M. Grioni, Rev. Sci. Instrum. 77, 113108 (2006). 